\title{
Judgment of spatial orientation in patients with focal brain damage ${ }^{1}$
}

\author{
ENNIO DE RENZI², PIERO FAGLIONI, AND GIUSEPPE SCOTTI
}

From the Clinic for Nervous and Mental Disease, University of Trieste and the Clinic for Nervous and Mental Disease, University of Milan, Italy

SUMMARY Thirty control and 121 brain-damaged patients with injury restricted to one hemisphere were presented with a test requiring the placing of a rod, fixed on a support by a hinged joint, in the same position as a model. Two versions of the test were given, one to be performed with the aid of vision and the other only by touch. The brain-damaged patients were subdivided into the following groups : right hemisphere patients without visual field defect (VFD): 30; right hemisphere patients with VFD: 20; left hemisphere patients without VFD: 46; left hemisphere patients with VFD: 25. The test modality did not differentially affect the performance of the groups. Both on the visually and on the tactually guided version of the test the right hemisphere group with VFD did significantly worse not only with respect to controls but also with respect to the three other braindamaged groups. The latter, in turn, were not significantly inferior to patients without cerebral damage. It appears, therefore, that when spatial perception is tested at a very basic and simple level, such as the detection of orientation in space of a rod, there is an almost complete dominance of the posterior region of the so-called minor hemisphere. This result must be contrasted with the less striking asymmetry of functions shown in more complex spatial tasks-for example, route finding, copying drawings, block designs-that are failed also by patients with damage to the left posterior area. Normal subjects reproduced the orientation of the rod on the horizontal plane with a constant error which was found to be dependent on the position of the model. Clockwise deviations were made when the model was on the left and counterclockwise deviations when the model was on the right. The constant error was present in left brain-damaged patients too, while it was not significantly different from zero in right brain-damaged patients. A systematic error was also found on the vertical plane and it consisted in the tendency to stop the rod below the level of the standard.

Findings derived from clinical case study and experimental research support the view that there is both a regional and a hemispheric specialization of the cerebral cortex for spatial functions in the sense that impairment in these functions is associated more closely with damage to posterior than to anterior areas of the brain and of the right more than of the left hemisphere. The evidence, however, is not entirely consistent. Some studies have substantiated the relevance of anteroposterior dimension, but not the hemispheric asymmetry (Semmes, Weinstein, Ghent, and Teuber, 1955; Colonna and

\footnotetext{
${ }^{1}$ This work was supported by grant no. 115.2348 .0 of the Consiglio Nazionale delle Ricerche.

- 2 Reprint requests: Professor De Renzi, Clinica delle Malattie Nervose e Mentali, Ospedale Maggiore, Trieste 34129, It aly.
}

Faglioni, 1966; Butters and Barton, 1970), while other researches have confirmed the crucial role of the right hemisphere but not the relevance of the left posterior region (Milner, 1965; Corkin, 1965; Newcombe and Russell, 1969). These apparent discrepancies are likely to be task dependent and they emphasize the need for analysing more carefully the nature of the performances, which have been defined as spatial. As a matter of fact, most of the tests used in neuropsychological investigation for assessing spatial abilities have been derived from intelligence tests or have been devised with specific neurological symptoms, such as constructional apraxia, topographical disorientation, or unilateral inattention in mind. Consequently, space perception has been studied at a rather complex level, one at which it is difficult to disentangle the influence on performance 
of spatial as compared with praxic, intelligence, and memory factors. It seems reasonable to expect that a more definite answer to the issue concerning the relationship between locus of lesion and the breakdown of space functions might be obtained by employing elementary tasks, which tap the basic mechanisms underlying spatial perception. The appreciation of the direction in space of a segment can be assumed to be one of these mechanisms, in that it represents a basic step in building up several spatial performances, such as the reproduction of a drawing, which is carried out copying line after line, or the tactile recognition of shapes, which is achieved by reconstructing the successive changes of orientation made by the exploring hand (Semmes, 1965; De Renzi and Scotti, 1969). Impairment of this ability has been occasionally observed in the neurological literature and referred to as deformation of visual coordinates. In 1944 Lenz required 56 patients with missile injury to the brain to represent the horizontal, the vertical, and the sagittal coordinates with a stick held in their hands, while blindfolded. He singled out six 'positive' cases who, when required to place the stick in the horizontal or vertical position, showed rather consistent errors, because they tended to incline the top end of the vertical stick toward the right and the right end of the horizontal stick toward the top, thus forming an oblique angle. Two patients had right parietooccipital damage and four biparietal damage; in two of the latter cases the damage was more extensive in the right hemisphere. These findings, taken together with the lack of errors found in three patients with left parieto-occipital injury, lead Lenz to stress 'the great importance of the right parietooccipital cortical area for all directional operations in visual and sensorimotor space'.

In 1950 McFie, Piercy, and Zangwill described eight right brain-damaged patients with a predominant involvement of the occipito-parietal region, selected because they showed clear evidence of visual-spatial agnosia. In five cases the deformation of visual coordinates was tested by requiring the patient to represent the horizontal and the vertical with a stick held in one hand. Errors analogous to those reported by Lenz were found in three patients, displacement in a clockwise direction but without approximation of the axes was present in the fourth patient, while the performance was correct in the fifth case. These findings must be contrasted with those reported successively by McFie and Zangwill (1960) in eight patients with damage to the left posterior parietal region, who showed visualconstructive impairment. The deformation of coordinates was tested in five of these patients and found to be absent in all of them.
The ability of patients to perceive the directiond of tactile stimulation on their bodies has been inves-⿳亠二口犬 tigated by Carmon and Benton (1969). Patients with lesions of the right hemisphere showed a higho incidence of defective performance, while left brain-D damaged patients performed at an essentially normal level. No distinction was made in this study between anterior vs. posterior locus of the lesion.

There is, therefore, evidence suggesting a relation: between right-sided damage and impairment of the $\overrightarrow{\vec{F}}$ sense of direction in space, even when this is studief 6 at its most elementary level. In the present investiga 등 tion it was hoped to gain more definite information $\overline{\bar{s}}$. on this topic by refining assessment of performancep and by collecting data on an unselected series of patients. Visually and tactually guided versions of the same test were given with the purpose of deter- $\overrightarrow{0}$ mining whether performance was dependent upon visuoperceptual and oculomotor mechanisms or ${ }^{\omega}$ whether it was supramodally organized.

\section{METHODS}

PATIENTS The patients examined in the present researes were the same as those described in a previous pare (De Renzi and Scotti, 1969), to which the readerबis referred for information regarding criteria adopted $f^{\prime}$ diagnosing the side of the lesion and the presence $\$ \mathrm{f}-$ visual field defects (VFD). One-hundred-and-twenty patients with damage restricted to one hemisphere werreे assigned to one of the following subgroups: (1) hemisphere patients without VFD (46); (2) left hemisphere patients with VFD (25); (3) right hemisphefe patients without VFD (30); (4) right hemisphere patients with VFD (20). The proportion of patients suffering from quadrantanopia and hemianopia was not found too be different in the two hemispheric groups.

The aetiology and the length of illness were determinedo and found not to differ significantly in the four sub-0 groups. No effort was made to equate the groups with respect to age and years of schooling, since the effect of these two variables was partialled out by covariance? analysis.

Thirty patients hospitalized in the wards for disease not involving the brain made up the control group.

TESTING PROCEDURE Both the visual and the tactile tests were carried out by the brain-damaged patients using only the hand homolateral to the cerebral side of the lesion-that is, the hand subserved by the supposedly healthy hemisphere. Fifteen control patients performed the tests with the right hand and 15 with the left hand.

Figure 1 shows the apparatus that was designed for and used in the study as a test of perception of spatial cor ordinates. Two pairs of rods, $38 \mathrm{~cm}$ apart, were fixed on? a board, $55 \mathrm{~cm} \times 15 \mathrm{~cm}$. Each pair was made up of a vertical rod, which could rotate $360^{\circ}$ round its axis, ando of a second rod, which was fixed to the first by a hinged joint in such a way that it could pivot up and down in the sagittal plane. By appropriate rotation of the two rods, $\omega$ 


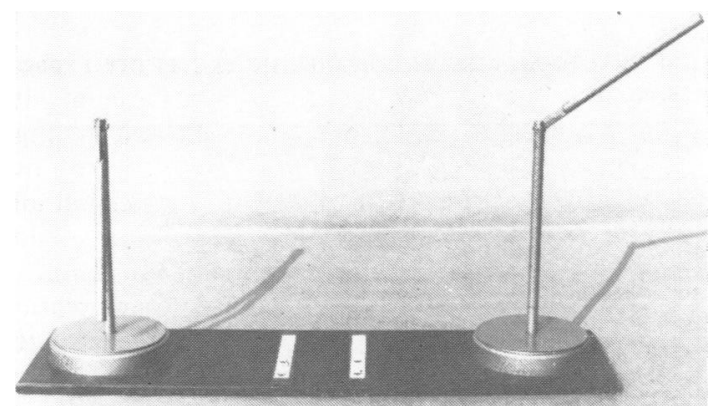

FIG. 1. The rod orientation test. Model to the right, copy to the left.

the second rod could be placed in any position in space that corresponded to the radii of an ideal sphere, having its centre in the joint between the two rods.

The patient was seated in front of the apparatus and was permitted to move his head and eyes but not his trunk. One pair of rods represented the model and the patient was asked to set the rods of the other pair to correspond to the standard.

There were two versions of the test, given through the visual and the tactile modalities, respectively. In the visual test, the patient was not permitted to touch the rods of the model and was requested to set those of the other pair using the hand homolateral to the side of the cerebral lesion. In the tactile test the apparatus was hidden behind a curtain and the patient had to estimate the spatial position of the rods of the model and of those of the copy by palpating them with the hand homolateral to the side of the lesion. No time limit was set and the patient was permitted to go from the model to the copy and vice versa as many times as he wished. The order of presentation of the two tests was alternated from patient to patient.

Both in the visual and in the tactile version of the test, brain-damaged patients were first given five trials with the model placed on the side ipsilateral to the damaged hemisphere and then five trials with the model on the contralateral side. Half of the control subjects began with the model to their left while the other half began with the model to their right. In each trial the spatial orientation of the model was determined by measuring with a goniometer: (1) the vertical angle of the two rods. It was agreed that the maximum width of this angle corresponded to the position in which the two rods were on the same vertical axis-that is, the angle could not exceed $180^{\circ} ;(2)$ the horizontal angle made by the second rod with respect to the sagittal plane, the zero point being just in front of the patient's midline. This angle could range, therefore, from $0^{\circ}$ to $360^{\circ}$ (see Fig. 1). The spatial parameters of the model in the five trials were as follows: 1 st trial $=\mathrm{v}($ ertical $)$ a(ngle): $90^{\circ}$; $\mathrm{h}$ (orizontal) $\mathrm{a}($ ngle $): 180^{\circ}$. 2nd trial $=$ v.a.: $45^{\circ}$; h.a.: $90^{\circ}$. 3rd trial $=$ v.a.: $130^{\circ}$; h.a.: $320^{\circ}$. 4th trial =v.a.: $60^{\circ}$; h.a.: $160^{\circ}$. 5 th trial $=$ v.a.: $150^{\circ}$; h.a.: $230^{\circ}$. At the beginning of each trial the position of the rods to be adjusted was always the same-namely, with the second rod pivoted down on the first rod and facing the patient (see Fig. 1).

\section{RESULTS}

Each copying performance was evaluated by measuring with a goniometer the deviations from the model in either directions of the vertical angle and the horizontal angle. Since the maximum possible width of the horizontal angle was twice that of the vertical angle, horizontal scores were divided by two. The scores obtained when the model was on the left side were treated separately from those obtained when the model was on the right. Thus there were four mean scores (horizontal and vertical angle with the model on the left and on the right) for both the visual and the tactile test.

These eight mean scores were submitted to a multivariate analysis of co-variance (Anderson, 1958), concomitant variables being age and years of schooling. A split-plot design was adopted. There were two groups, the control and the brain-damaged groups, which were further subdivided, the first into two subgroups (subjects using the left hand and the right hand, respectively) and the second into four subgroups (left and right hemisphere patients with and without VFD). In this experimental design three hypotheses, orthogonal to each other, could be tested, using Wilks's criterion (1932) and Rao's approximation (1951) to the $\mathrm{F}$ distribution. These hypotheses were concerned with the question of whether the set of the eight scores differentiated the performance of: (a) the control and the braindamaged group (hypothesis 1); (b) the two control subgroups (hypothesis 2); and (c) the four braindamaged subgroups (hypothesis 3 ).

Table 1 presents the mean error scores (in degrees) obtained by the two control and four brain-damaged subgroups in the eight experimental conditions and Table 2 shows the results of the multivariate analysis. Let us consider first the second hypothesis, which deals with the question whether the performance of control subjects was affected by the hand used in carrying out the test. Since the corresponding $\mathbf{P}$ fell short of the 0.05 significance level, the conclusion seems safe that any difference to be found in subsequent comparisons cannot be attributed to the hand effect.

By the first multivariate hypothesis the set of the eight means was found to differentiate the control and the brain-damaged group beyond the 0.05 level of significance (one-tailed test). With the multiple compar ison method of Roy and Bose (1953) it was found that neither the test modality, the position 
TABLE 1

MEAN ERRORS (IN DEGREES) OF THE TWO CONTROL AND THE FOUR BRAIN-DAMAGED SUBGROUPS IN THE EIGHT EXPERIMENTAL CONDITIONS

\begin{tabular}{|c|c|c|c|c|c|c|c|c|}
\hline & \multicolumn{4}{|c|}{ Visual test } & \multicolumn{4}{|c|}{ Tactile test } \\
\hline & \multicolumn{2}{|c|}{ Horizontal } & \multicolumn{2}{|c|}{ Vertical } & \multicolumn{2}{|c|}{ Horizontal } & \multicolumn{2}{|c|}{ Vertical } \\
\hline & $\begin{array}{c}\text { M. on } \\
\text { left }\end{array}$ & $\begin{array}{c}\text { M. on } \\
\text { right }\end{array}$ & $\begin{array}{c}\text { M. on } \\
\text { left }\end{array}$ & $\begin{array}{c}\text { M. on } \\
\text { right }\end{array}$ & $\begin{array}{c}\text { M. on } \\
\text { left }\end{array}$ & $\begin{array}{c}\text { M. on } \\
\text { right }\end{array}$ & $\begin{array}{c}\text { M. on } \\
\text { left }\end{array}$ & $\begin{array}{c}\text { M. on } \\
\text { right }\end{array}$ \\
\hline \multicolumn{9}{|l|}{ Controls } \\
\hline Left hand & 7 & 6 & 4 & 4 & 6 & 6 & 8 & 8 \\
\hline Right hand & 4 & 6 & 4 & 5 & 7 & 7 & 6 & 8 \\
\hline \multicolumn{9}{|l|}{ Hemisphere } \\
\hline Left, VFD - & 5 & 5 & 5 & 5 & 7 & 7 & 9 & 9 \\
\hline Left, VFD + & 6 & 6 & 5 & 7 & 8 & 9 & 9 & 8 \\
\hline Right, VFD - & 6 & 6 & 5 & 6 & 7 & 8 & 8 . & 9 \\
\hline Right, VFD + & 8 & 9 & 9 & 9 & 10 & 12 & 13 & 18 \\
\hline
\end{tabular}

TABLE 2

MULTIVARIATE ANALYSIS OF COVARIANCE

\begin{tabular}{lccc}
\hline \multicolumn{1}{c}{ Comparison } & D.F. & $F$ & $P$ \\
\hline $\begin{array}{l}\text { Control } v s . \text { brain-damaged patients } \\
\text { (hypothesis 1) }\end{array}$ & $8 / 136$ & 1.715 & $<0.05^{*}$ \\
$\begin{array}{l}\text { Subgroups within controls } \\
\text { (hypothesis 2) }\end{array}$ & $8 / 136$ & 1.346 & n.s. \\
$\begin{array}{l}\text { Subgroups within brain-damaged patients } \\
\text { (hypothesis 3) }\end{array}$ & $24 / 395$ & 2.884 & $<0.001 \dagger$ \\
\hline
\end{tabular}

* = one-tailed test.

$\dagger=$ two-tailed test

of the model, the two angular scores, nor any interaction among these factors significantly affected the intergroup difference.

The outcome of the third multivariate hypothesis showed that there was a highly significant difference (P $<0.0001)$ among the performance of the four brain-damaged subgroups, a finding that inspection of the means of Table 1 suggests to be due mainly to the elective impairment of the right hemisphere subgroup with VFD. In order to verify this point, the scores of the four brain-damaged subgroups and the combined score of the control group were compared with each other by the multiple comparison method of Roy and Bose (1953). Since, at the level of the third multivariate hypothesis, no interaction of a first or higher order with testing modality, the position of the model and the angular score were found significantly to affect the intergroup difference, the multiple comparisons were carried out on the means and not on the set of the eight scores obtained by each group. These means are represented in Fig. 2. The multiple comparison method yields theta values, which must be compared with the upper percentage points of the generalized beta dis- tributions (theta points). The theta values obtained $\stackrel{\circ}{=}$ for each comparison and the theta points corresponding to the 0.05 and 0.01 probability level are given in Table 3. It is apparent that right hemisphere + patients with VFD were significantly inferior to the other groups, while the performance of the other brain-damaged subgroups did not significantfy differ from each other and also with respect to t controls' performance.

Since the primary interest of this study was hin precision of spatial orientation with respect to standard, the statistic used was the mean of the ut signed deviations from the position of the mode $\vec{\theta}$ However, in the course of the research, it was noted.

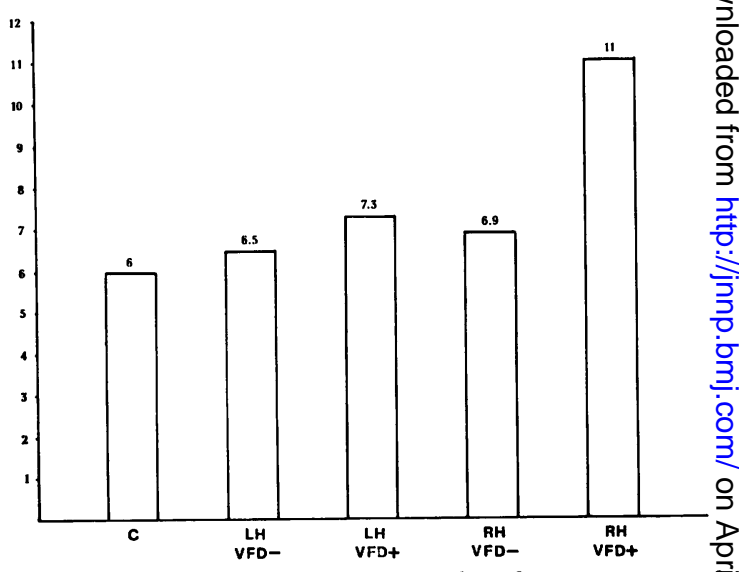

FIG. 2. Columns represent the combined mean scores of controls $(C)$, left hemisphere patients without VFD $(L H, \mathrm{~N}$ $V F D-)$, left hemisphere patients with $V F D(L H, V F D+), N$ right hemisphere patients without VFD (RH,VFD-) ) right hemisphere patients with VFD (RH.VFD+). 
TABLE 3

THETA VALUES OF THE MULTIPLE COMPARISONS

\begin{tabular}{lcccc}
\hline & $L H, V F D-$ & $L H, V F D+$ & $R H, V F D-$ & $R H, V F D+$ \\
\hline Controls & -0.0028 & -0.0162 & -0.114 & $-0.2305 \dagger$ \\
LH, VFD - & & -0.0089 & -0.0042 & $-0.2384 \dagger$ \\
LH, VFD + & & & -0.0010 & $-0.1438^{*}$ \\
RH, VFD - & & & & $-0.1790 \dagger$ \\
\hline
\end{tabular}

Theta points with parameters $1,144,4: \mathrm{p}_{05}=0.0979 ; \mathrm{p}_{01}=0.1592$. ${ }^{*} \mathrm{P}<0.05$. $\quad+\mathrm{P}<0.01$.

that there was a tendency for many patients to make a constant error in the horizontal plane, depending on the side of presentation of the model. It was decided, therefore, to verify this impression by measuring the algebraical mean of the deviations of the rod with respect to the standard. Two separate analyses were carried out, one on the horizontal scores and the other on the vertical scores. Table 4 presents the mean signed deviations of the horizontal scores of the control group and of the four brain-damaged subgroups for the two versions of the test and for the left and the right position of the model. Clockwise deviations are designed as positive $(+)$ and counterclockwise deviations as negative $(-)$. It is apparent that the deviations were clockwise when the model was on the left and counterclockwise when the model was on the right. A four-variate analysis of covariance (age and years of schooling being the independent variables) showed that the five sets of the four scores were significantly different from zero $(\mathrm{F}=9.92 ; \mathrm{P}<$ 0.0001 with DF 20 and 468). Multiple comparisons confirmed that the left-right gradient-that is, the difference between the sum of the signed deviations with the model on the left and the sum of the signed deviations with the model on the rightwas highly significant for the total sample of patients $(P<0.01)$. When tested in each of the five groups, the gradient proved to be significantly different from zero in the control group and in the two left braindamaged subgroups, but not in the two right braindamaged subgroups, suggesting that lesions of the

TABLE 4

MEANS IN DEGREES OF CONSTANT ERROR MADE BY FIVE GROUPS IN HORIZONTAL PLANE

\begin{tabular}{lccccc}
\hline & \multicolumn{2}{c}{ Model on left } & & \multicolumn{2}{c}{ Model on right } \\
\cline { 2 - 3 } \cline { 5 - 6 } & Visual & Tactile & & Visual & Tactile \\
\hline Controls & +5 & +3 & & -4 & -2 \\
LH, VFD - & +4 & +4 & & -2 & -3 \\
LH, VFD + & +5 & +4 & & -4 & -2 \\
RH, VFD - & +3 & +2 & & -1 & -1 \\
RH, VFD + & +2 & +0 & & -3 & -1 \\
\hline
\end{tabular}

right hemisphere tend to lower the constant error. No significant difference in the size of the gradient was found, however, in any inter-group comparison carried out. Neither the test modality nor the modality $\mathrm{x}$ model position interaction affected the constant error.

With respect to the vertical score, Table 5 shows that in both test modalities and model positions there was a tendency to stop the rod below the level of the standard. When tested with the same analysis used for the horizontal score this error was highly significant $(\mathrm{P}<0.0001)$ in the total sample of patients and its size did not change significantly from one group to the other.

\section{TABLE 5}

MEANS (IN DEGREES) OF CONSTANT ERROR MADE BY FIVE GROUPS IN VERTICAL PLANE

\begin{tabular}{lccccc}
\hline & \multicolumn{2}{c}{ Model on left } & & \multicolumn{2}{c}{ Model on right } \\
\cline { 2 - 3 } \cline { 5 - 6 } & Visual & Tactile & & Visual & Tactile \\
\hline Controls & -1 & -3 & & -2 & -3 \\
LH, VFD - & -1 & -2 & & -2 & 0 \\
LH, VFD + & -2 & -2 & & -2 & 0 \\
RH, VFD - & 0 & -2 & & -4 & -4 \\
RH, VFD + & -1 & -4 & & 0 & -4 \\
\hline
\end{tabular}

\section{DISCUSSION}

In the present as well as in previous researches carried out in our laboratory the hemispheric samples have been subdivided according to the absence or presence of VFD, because it was thought that this easily detectable symptom could provide useful information regarding the intra-hemispheric locus of lesion. The central optic pathways run in the white matter underlying the border between the temporal and the parietal lobe and end in the calcarine fissure of the occipital lobe; therefore, any lesion localized in front of the Rolandic fissure should spare the optic radiations, while retroRolandic injuries are likely to produce hemi- or quadrantanopia. It must be recognized that this dichotomy is rough and imprecise, since, for example, superior parietal lesions do not involve optic pathways and, consequently, the corresponding patients would be considered together with those suffering from pre-Rolandic damage. This drawback can, however, be overlooked whenever significant differences are found between the performance of groups with and without VFD, because mutual overlap of their lesions with respect to the Rolandic sulcus would diminish rather than enhance the probability of finding differential results.

Before accepting the conclusion that impairment 
associated with VFD indicates the detrimental effect on performance produced by damage to retroRolandic areas, the possibility must be ruled out that the impairment simply reflects the deficit of visual functions. In the results of our experiment there are at least two pieces of evidence that argue against this possibility. Firstly, the group that fails on the visual test also fails on the tactile test, which is carried out without the aid of vision. Secondly, apparently the presence of VFD does not impair performance when it is associated with left hemisphere lesions, but only when it is associated with right hemisphere lesions, a finding which points to the importance of the locus of brain injury. On the basis of these considerations, we deem it justified to assume that damage to the retro-Rolandic region of the right hemisphere plays a unique role in deranging such an elementary ability as that implied by the evaluation of the spatial direction of a rod. The fact that none of the other brain-damaged subgroups, including that with retro-Rolandic injury to the left hemisphere, was found to be impaired with respect to control patients is worth emphasizing, because some previous researches have indicated a decrement in spatial performance after injury to either of the two posterior areas of the brain, although more severely when the right one was involved.

It is noteworthy that lack of impairment in left posterior brain-damaged patients has been reported, to the best of our knowledge, only for the learning of maze tests. Whether given visually (Milner, 1965; Newcombe and Russell, 1969) or tactually (Corkin, 1965), they have demonstrated disruption of the performance in comparison with normals after right, but not after left hemispheric injury. Appreciation of spatial orientation is a prominent feature of these tests, although there has been discussion to what extent the critical variable in determining the patient's failure is learning or spatial perception. The first hypothesis is supported by the finding that in right temporal lobectomized patients the ablation of a major part of the hippocampus appeared to be decisive in lowering performance (Milner, 1965; Corkin, 1965). On the other hand, Newcombe and Russell (1969) presented evidence that poor scores on the maze test were associated with right parietal but not with right temporal lobe damage and that they correlated positively with the scores of other spatial perceptual tests. While it is possible that patients with differently localized lesions fail for different reasons, our results parallel those of Newcombe and Russell in showing the existence of a true right hemisphere dominance in the perceptual processing of spatial clues. These findings must be contrasted with those obtained from other maze tests, or similar tests, which assess extrapersonal orientation at a level involving intelligence, as in the on Elithorn's maze test (Elithorn, 1955) or the translation of perceptual informations into complex locomotor behaviour, as in the route finding test described by Semmes et al. (1955). In these cases right $\stackrel{?}{ }$ hemisphere dominance shifts towards asymmetry of functions (Benton, Elithorn, Fogel, and Kerr, 1963), .. or equipotentiality of the two posterior regions $\overrightarrow{\vec{F}}$ (Semmes et al., 1955). The close association between $\stackrel{?}{\rightarrow}$ poor scores and language disorders found both in the Elithorn test (Colonna and Faglioni, 1966) and $\frac{\overline{\bar{c}}}{\mathrm{~s}}$ in the route finding test (Semmes, Weinstein, Ghent, $\frac{\sigma}{\overrightarrow{0}}$ and Teuber, 1963) may be interpreted as evidence $\varrho$ that the impairment shown by left brain-damaged $\%$ patients is due to the lack of the aid provided to the $\overrightarrow{0}$ performance by verbal mediation. The alternative and broader view may be advanced that the con- $\vec{\omega}$ tribution of the left hemisphere to 'pure' spatial perception is minimal or nil, whereas it increases more and more as the task increasingly requires the elaborate integration of spatial data or the grasp- + ing of their mutual relationships.

The lack of impairment of space perception foun in the present research after brain damage not 0 involving the right posterior region is all the mo remarkable as most of our patients were suffering 0 from recent and considerably incapacitating disease, so that it seems unlikely that good performan may be attributed to restoration of function. On the other hand, it must be recognized that the vascul $\overrightarrow{0}$ aetiology prevailing in our case material does not $\stackrel{-}{-}$ often produce damage to the prefrontal areas and it $\square$ is, therefore, open to question to what extent the negative results found in patients without VFD are valid also for the involvement of this area of the brain. This issue deserves further investigation. For $\frac{\circ}{D}$ the time being, it seems safe to stress the point that, $\stackrel{\varrho}{\Rightarrow}$ while complex tasks may be more sensitive in disclosing mild disorders of spatial orientation, as suggested by Milner (1965) in comparing her results with those of Semmes et al. (1955), elementary perceptual tasks provide a more suitable method $\overline{5}$ for bringing out hemispherical differences in? function.

The lack of differential impairment found in the visual and the tactile version of the test confirms $O$ previous findings (Semmes et al., 1955; Corkin, $₹$ 1965; Faglioni, Scotti, and Spirnler, 1969; De Renzi, 음 Faglioni, and Scotti, 1970) that extrapersonal dis- $>$ orientation is not restricted to vision and that the processing of spatial clues is supramodally organ- $N$ ized. The available evidence points to the right ${ }^{\circ}$ parietal lobe as the crucial area involved in this 0 function.

The constant error related to the position of the 
model that control subjects made in reproducing the orientation of the rod on the horizontal plane is not comparable with the error reported in orientation tasks, involving the setting of a luminous line to the apparent vertical (Aubert phenomenon). In that experimental situation the constant error was found only while the body was tilted and was minimal or nil while the body was upright (Teuber and Liebert, 1958; Wapner and Werner, 1965), whereas the constant error we observed does not need conditions of abnormal body posture to be brought out. Neither does it depend on visual factors, since the magnitude of the error is not significantly different in the visual and in the tactile modality of the test. Further investigation is needed to clarify the nature of this error, all the more so as the experimental conditions of the present research were not favourable to its analysis: in the five trials, the horizontal angles of the model were not alike when the second rod was to the left and when it was to the right of the sagittal plane and they were made even more difficult to compare by the various orientations of the rod in the vertical field.

Nevertheless, the fact that lesions of the right hemisphere-that is, of the hemisphere specialized in processing spatial clues-lower the constant error to the point that it is no more significantly different from zero deserves consideration. Although the difference with respect to either the left braindamaged patients or the controls falls short of the accepted level of confidence, it is worth contrasting this finding with changes in the size of the constant error reported by researches on the judgment of the vertical line. With body upright-that is, in the experimental condition more alike to the one examined in the present investigation-the magnitude of the constant error of brain-damaged patients was found significantly increased with respect to controls (Bender and Jung, 1948), or practically unchanged (Teuber and Mishkin, 1954; Birch, Belmont, Reilly, and Belmont, 1961), but never decreased. It must be remembered, however, that with the body upright, the constant error is very mild or absent in control subjects required to set a luminous line to the vertical, while it is a distinctive feature of the normals' performance in our experimental condition. It is, therefore, not surprising that it may be depressed by damage to the cerebral hemisphere which is specialized in spatial orientation.

\section{REFERENCES}

Anderson, T. W. (1958). Introduction to Multivariate Statistical Analysis. Wiley: New York.

Bender, M., and Jung, R. (1948). Abweichungen der subjectiven optischen Vertikalen und Horizontalen bei Gesunden und Hirnverletzten. Arch. Psychiat. Nervenkr., 181, 193212.
Benton, A. L., Elithorn, A., Fogel, M. L., and Kerr, M. (1963). A perceptual maze test sensitive to brain damage. J. Neurol. Neurosurg. Psychiat., 26, 540-544.

Birch, H. G., Belmont, I., Reilly, T., and Belmont, L. (1961). Visual verticality in hemiplegia. Visual influences on perception. Arch. Neurol. (Chic.), 5, 444-453.

Butters, N., and Barton, M. (1970). Effect of parietal lobe damage on the performance of reversible operations in space. Neuropsychologia, 8, 205-214.

Carmon, A., and Benton, A. L. (1969). Tactile perception of direction and number in patients with unilateral cerebral disease. Neurology (Minneap.), 19, 525-532.

Colonna, A., and Faglioni, P. (1966). The performance of hemisphere-damaged patients on spatial intelligence tests. Cortex, 2, 293-307.

Corkin, S. (1965). Tactually-guided maze learning in man: effects of unilateral cortical excisions and bilateral hippocampal lesions. Neuropsychologia, 3, 339-351.

De Renzi, E., Faglioni, P., and Scotti, G. (1970). Hemispheric contribution to exploration of space through the visual and tactile modality. Cortex, 6, 191-203.

De Renzi, E., and Scotti, F. (1969). The influence of spatial disorders in impairing tactual discrimination of shapes. Cortex, 5, 53-62.

Elithorn, A. (1955). A preliminary report on a perceptual maze test sensitive to brain damage. J. Neurol. Neurosurg. Psychiat., 18, 287-292.

Faglioni, P., Scotti, G., and Spinnler, H. (1969). Asimmetria emisferica nella localizzazione spaziale di stimoli visivi e tattili Arch. Psicol. Neurol. Psichiat., 30, 513-525.

Lenz, H. (1944). Raumsinnstörungen bei Hirnverletzungen. Disch. Z. Nervenheilk., 157, 22-64.

McFie, J., Piercy, M. F., and Zangwill, O. L. (1950). Visualspatial agnosia associated with lesions of the right cerebral hemisphere. Brain, 73, 167-190.

McFie, J., and Zangwill, O. L. (1960). Visual constructive disabilities associated with lesions of the left cerebral hemisphere. Brain, 83, 243-260.

Milner, B. (1965). Visually-guided maze learning in man: effects of bilateral hippocampal, bilateral frontal, and unilateral cerebral lesions. Neuropsychologia, 3, 317-338.

Newcombe, F., and Russell, W. R. (1969). Dissociated visual perceptual and spatial deficits in focal lesions of the right hemisphere. J. Neurol. Neurosurg. Psychiat., 32, 73-81.

Rao, C. R. (1951). An asymptotic expansion of the distribution of Wilks ${ }^{-} \mathrm{X}$ criterion. Bull. int. Statist. Inst., 33, Part 2, 177-180.

Roy, S. N., and Bose, R. C. (1953). Simultaneous confidence interval estimation. Ann. math. Statist., 24, 513-536.

Semmes, J. (1965). A non-tactual factor in astereognosis. Neuropsychologia, 3, 295-315.

Semmes, J., Weinstein, S., Ghent, L., and Teuber, H.-L. (1955). Spatial orientation in man after cerebral injury. I. Analyses by locus of lesion. J. Psychol., 39, 227-244.

Semmes, J., Weinstein, S., Ghent, L., and Teuber, H.-L. (1963). Correlates of impaired orientation in personal and extrapersonal space. Brain, 86, 747-772.

Teuber, H.-L., and Liebert, R. S. (1958). Specific and general effects of brain injury in man. Evidence of both from a single task. Arch. Neurol. Psychiat. (Chic.), 80, 403-407.

Teuber, H.-L., and Mishkin, M. (1954). Judgment of visual and postural vertical after brain injury. J. Psychol., 38, 161-175.

Wapner, S., and Werner, H. (1965). An experimental approach to body perception from the organismic-developmental point of view. In The Body Percept, pp. 12-16. Edited by $\mathrm{S}$. Wapner and $\mathrm{H}$. Werner. Random House: New York.

Wilks, S. S. (1932). Certain generalizations in the analysis of variance. Biometrika, 24, 471-494. 